\title{
On some properties of representation functions related to the Erdős-Turán conjecture
}

\author{
Csaba Sándor ${ }^{1 *}$ Quan-Hui Yang ${ }^{2 \dagger}$ \\ 1. Institute of Mathematics, Budapest University of Technology and Economics, H-1529 B.O. \\ Box, Hungary \\ 2. School of Mathematics and Statistics, Nanjing University of Information \\ Science and Technology, Nanjing 210044, China
}

\begin{abstract}
For a set $A \subseteq \mathbb{N}$ and $n \in \mathbb{N}$, let $R_{A}(n)$ denote the number of ordered pairs $\left(a, a^{\prime}\right) \in$ $A \times A$ such that $a+a^{\prime}=n$. The celebrated Erdős-Turán conjecture says that, if $R_{A}(n) \geq 1$ for all sufficiently large integers $n$, then the representation function $R_{A}(n)$ cannot be bounded. For any positive integer $m$, Ruzsa's number $R_{m}$ is defined to be the least positive integer $r$ such that there exists a set $A \subseteq \mathbb{Z}_{m}$ with $1 \leq R_{A}(n) \leq r$ for all $n \in \mathbb{Z}_{m}$. In 2008, Chen proved that $R_{m} \leq 288$ for all positive integers $m$. Recently the authors proved that $R_{m} \geq 6$ for all integers $m \geq 36$. In this paper, for an abelian group $G$, we prove that if $A \subseteq G$ satisfies $R_{A}(g) \leq 5$ for all $g \in G$, then $\left|\left\{g: g \in G, R_{A}(g)=0\right\}\right| \geq \frac{1}{4} m-\sqrt{5 m}$. This improves a recent result of Li and Chen. We also give upper bounds of $\left|\left\{g: g \in G, R_{A}(g)=i\right\}\right|$ for $i=2,4$.

2010 Mathematics Subject Classification: Primary 11B34,11B13.

Keywords and phrases: Representation function, Ruzsa's number, Erdős-Turán conjecture
\end{abstract}

\section{Introduction}

Let $G$ be an abelian group. For any set $A, B \subseteq G$, let

$$
R_{A, B}(g)=\sharp\{(a, b): a \in A, b \in B, a+b=g\} .
$$

\footnotetext{
*Email: csandor@math.bme.hu. This author was supported by the OTKA Grant No. K109789. This paper was supported by the János Bolyai Research Scholarship of the Hungarian Academy of Sciences.

$\dagger$ Email: yangquanhui01@163.com. This author was supported by the National Natural Science Foundation for Youth of China, Grant No. 11501299, the Natural Science Foundation of Jiangsu Province, Grant Nos. BK20150889, 15KJB110014 and the Startup Foundation for Introducing Talent of NUIST, Grant No. $2014 \mathrm{r} 029$.
} 
Let $R_{A}(g)=R_{A, A}(g)$. If $A \subseteq \mathbb{N}$ and $R_{A}(n) \geq 1$ for all sufficiently large integers $n$, then we say that $A$ is a basis of $\mathbb{N}$. The celebrated Erdős-Turán conjecture [7] states that if $A$ is a basis of $\mathbb{N}$, then $R_{A}(n)$ cannot be bounded. Erdös [6] proved that there exists a basis $A$ and two constants $c_{1}, c_{2}>0$ such that $c_{1} \log n \leq R_{A}(n) \leq c_{2} \log n$ for all sufficiently large integers $n$. Recently, Dubickas [5] gave the explicit values of $c_{1}$ and $c_{2}$. In 2003, Nathanson [15] proved that the Erdős-Turán conjecture does not hold on $\mathbb{Z}$. In fact, he proved that there exists a set $A \subseteq \mathbb{Z}$ such that $1 \leq R_{A}(n) \leq 2$ for all integers $n$. In the same year, Grekos et al. 8] proved that if $R_{A}(n) \geq 1$ for all $n$, then $\lim \sup _{n \rightarrow \infty} R_{A}(n) \geq 6$. Later, Borwein et al. [1 improved 6 to 8. In 2013, Konstantoulas [1] proved that if the upper density $\bar{d}(\mathbb{N} \backslash(A+A))$ of the set of numbers not represented as sums of two numbers of $A$ is less than $1 / 10$, then $R_{A}(n)>5$ for infinitely many natural numbers $n$. Chen 3 . proved that there exists a basis $A$ of $\mathbb{N}$ such that the set of $n$ with $R_{A}(n)=2$ has density one. Later, the second author [20] and Tang [19] generalized Chen's result. For the analogue of Erdős-Turán conjecture in groups, one can refer to [9], [10] and [12].

For a positive integer $m$, let $\mathbb{Z}_{m}$ be the set of residue classes $\bmod m$. If $R_{A}(n) \geq 1$ for all $n \in \mathbb{Z}_{m}$, then $A$ is called an additive basis of $\mathbb{Z}_{m}$.

In 1990, Ruzsa [16] found a basis $A$ of $\mathbb{N}$ for which $R_{A}(n)$ is bounded in the square mean. Ruzsa's method implies that there exists a constant $C$ such that for any positive integer $m$, there exists an additive basis $A$ of $\mathbb{Z}_{m}$ with $R_{A}(n) \leq C$ for all $n \in \mathbb{Z}_{m}$. For each positive integer $m$, Chen [2] defined Ruzsa's number $R_{m}$ to be the least positive integer $r$ such that there exists an additive basis $A$ of $\mathbb{Z}_{m}$ with $R_{A}(n) \leq r$ for all $n \in \mathbb{Z}_{m}$. In the same paper, Chen proved that $R_{m} \leq 288$ for all positive integer $m$ and $R_{2 p^{2}} \leq 48$ for all primes $p$.

In 2016, the authors [17]. proved that if $m \geq 36$, then $R_{m} \geq 6$. That is, if $m \geq 36$ and $A \subseteq \mathbb{Z}_{m}$ satisfies $R_{A}(n) \leq 5$ for all integers $n$, then there exists a $n_{0} \in \mathbb{Z}_{m}$ such that $R_{A}\left(n_{0}\right)=0$. Recently, Li and Chen (see [14, Corollary 1.3]) gave a quantitative version of this result.

Li \& Chen's Theorem. Let $G$ be a finite abelian group with $|G|=m$ and $A \subseteq G$. If $R_{A}(g) \leq 5$ for all $g \in G$, then

$$
\left|\left\{g: g \in G, R_{A}(g)=0\right\}\right| \geq \frac{7}{32} m-\frac{1}{2} \sqrt{10 m}-1 .
$$

In this paper, we improve $\mathrm{Li}$ and Chen's theorem and also give an example on the other hand. For convenience, for a fixed nonnegative integer $i$, we denote the set $\{g: g \in$ $G, R_{A}(g)=i$ ) by $S_{i}$.

Theorem 1. (a) Let $G$ be a finite abelian group with $|G|=m$ and $A \subseteq G$. If $R_{A}(g) \leq 5$ for all $g \in G$, then $\left|S_{0}\right| \geq \frac{1}{4} m-\sqrt{5 m}$.

(b) Let $p$ be a prime and $m=2\left(p^{2}+p+1\right)$. Then there exists a subset $A \subseteq \mathbb{Z}_{m}$ such that $R_{A}(n) \leq 5$ for all $n \in \mathbb{Z}_{m}$ and $\left|S_{0}\right|<\frac{3}{8} m$. 
If $R_{A}(g) \leq 5$ for all $g \in G$, then by $\left|S_{0}\right|+\left|S_{2}\right|+\left|S_{4}\right| \leq m$ and Theorem 1 (a), we see that $\left|S_{2}\right|+\left|S_{4}\right| \leq \frac{3}{4} m+\sqrt{5 m}$. In the next two theorems, we give upper bounds for $\left|S_{2}\right|$ and $\left|S_{4}\right|$ respectively.

Theorem 2. (a) Let $A \subseteq G$ satisfy $R_{A}(g) \leq 5$ for all $g \in G$. Then $\left|S_{2}\right| \leq \frac{1}{2} m+3 \sqrt{5 m}$.

(b) Let $p$ be a prime and $m=p^{2}+p+1$. Then there exists a subset $A \subseteq \mathbb{Z}_{m}$ such that $R_{A}(n) \leq 2$ for all $n \in \mathbb{Z}_{m}$ and $\left|S_{2}\right|=\frac{1}{2} m-\frac{1}{2}$.

Remark 1. The example in Theorem 回 (b) shows that Theorem 回 (a) is nearly best possible.

If $R_{A}(g) \leq 5$ for all $g \in G$, by the statement before Theorem 2, we have $\left|S_{2}\right|+\left|S_{4}\right| \leq$ $\frac{3}{4} m+\sqrt{5 m}$, and so $\left|S_{4}\right| \leq \frac{3}{4} m+O(\sqrt{m})$. It seems difficult to improve this upper bound. In the following, we will prove this result by a weak condition $R_{A}(g) \leq 7$ for all $g \in G$.

Theorem 3. (a) Let $A \subseteq G$ satisfy $R_{A}(g) \leq 7$ for all $g \in G$. Then $\left|S_{4}\right| \leq \frac{3}{4} m+O(\sqrt{m})$.

(b) Let $p$ be a prime and $m=2\left(p^{2}+p+1\right)$. Then there exists a subset $A \subseteq \mathbb{Z}_{m}$ such that $R_{A}(n) \leq 4$ for all $n \in \mathbb{Z}_{m}$ and $\left|S_{4}\right|=\frac{1}{2} m-1$.

\section{Preliminary Lemmas}

Lemma 1. (See [17, Lemma 3].) Let $A \subseteq G$ and $c$ be a positive integer. If $R_{A}(g) \leq c$ for all $g \in G$, then $|A| \leq \sqrt{\mathrm{cm}}$.

Lemma 2. (See [18, Singer's Theorem].) If $l$ is a prime power, then there exists $A \subseteq$ $\mathbb{Z}_{l^{2}+l+1}$ such that $R_{A,-A}(n)=1$ for all $n \in \mathbb{Z}_{l^{2}+l+1}, n \neq \overline{0}$.

Lemma 3. If $A$ is a subset of $G$, then for any positive integer $k$ we have

$$
\sum_{g \in G}\left(R_{A}(g)-k\right)^{2} \geq k m-(2 k-1)|A|+k^{2}-k .
$$

Proof. We use Lev and Sárközy's argument (see [13]) in the following.

$$
\begin{aligned}
\sum_{g \in G}\left(R_{A}(g)-k\right)^{2} & =\sum_{g \in G} R_{A}(g)^{2}-2 k \sum_{g \in G} R_{A}(g)+k^{2} m \\
& =\sum_{g \in G} R_{A,-A}(g)^{2}-2 k|A|^{2}+k^{2} m \\
& =\sum_{g \in G \backslash\{0\}} R_{A,-A}(g)^{2}-(2 k-1)|A|^{2}+k^{2} m \\
& \geq \frac{1}{m-1}\left(\sum_{g \in G \backslash\{0\}} R_{A,-A}(g)\right)^{2}-(2 k-1)|A|^{2}+k^{2} m \\
& =\frac{\left(|A|^{2}-|A|\right)^{2}}{m-1}-(2 k-1)\left(|A|^{2}-|A|\right)-(2 k-1)|A|+k^{2} m \\
& =(m-1)\left(\left(\frac{|A|^{2}-|A|}{m-1}-\left(k-\frac{1}{2}\right)\right)^{2}+k-\frac{1}{4}\right)-(2 k-1)|A|+k^{2} .
\end{aligned}
$$


If $\frac{|A|^{2}-|A|}{m-1} \geq k$ or $\frac{|A|^{2}-|A|}{m-1} \leq k-1$, then we have

$$
\sum_{g \in G}\left(R_{A}(g)-k\right)^{2} \geq k(m-1)-(2 k-1)|A|+k^{2}=k m-(2 k-1)|A|+k^{2}-k,
$$

and the result is true.

If $k-1<\frac{|A|^{2}-|A|}{m-1}<k$, then

$$
\sum_{g \in G \backslash\{0\}} R_{A,-A}(g)^{2} \geq \min _{\substack{k_{1}, k_{2}, \ldots, k_{m-1} \in \mathbb{N} \\ \sum_{i=1}^{m-1} k_{i}=|A|^{2}-|A|}} \sum_{i=1}^{m-1} k_{i}^{2} .
$$

It is known that if $\sum_{i=1}^{m-1} k_{i}$ is fixed, where $k_{i} \in \mathbb{N}$, then $\sum_{i=1}^{m-1} k_{i}^{2}$ gets the minimal value when $\left|k_{i}-k_{j}\right| \leq 1$ for all $1 \leq i, j \leq m-1$. Let $|A|^{2}-|A|=q(m-1)+r$, where $q, r$ are nonnegative integers and $0 \leq r<m-1$. Then $q=\left\lfloor\frac{|A|^{2}-|A|}{m-1}\right\rfloor$ and $r=\left\{\frac{|A|^{2}-|A|}{m-1}\right\}(m-1)$. Hence

$$
\begin{aligned}
\sum_{g \in G \backslash\{0\}} R_{A,-A}(g)^{2} & \geq \min _{\substack{k_{1}, k_{2}, \ldots, k_{m-1} \in \mathbb{N} \\
\sum_{i=1}^{m-1} k_{i}=|A|^{2}-|A|}} \sum_{i=1}^{m-1} k_{i}^{2}=r k+(m-1-r)(k-1) \\
& =\left\{\frac{|A|^{2}-|A|}{m-1}\right\}(m-1) k^{2}+\left(1-\left\{\frac{|A|^{2}-|A|}{m-1}\right\}\right)(m-1)(k-1)^{2} \\
& =(k-1)^{2}(m-1)+(2 k-1)\left\{\frac{|A|^{2}-|A|}{m-1}\right\}(m-1) .
\end{aligned}
$$

Therefore,

$$
\begin{aligned}
\sum_{g \in G}\left(R_{A}(g)-k\right)^{2}= & \sum_{g \in G \backslash\{0\}} R_{A,-A}(g)^{2}-(2 k-1)|A|^{2}+k^{2} m \\
\geq & (k-1)^{2}(m-1)+(2 k-1)\left\{\frac{|A|^{2}-|A|}{m-1}\right\}(m-1) \\
& -(2 k-1)\left(|A|^{2}-|A|\right)-(2 k-1)|A|+k^{2} m \\
= & (k-1)^{2}(m-1)-(2 k-1)(m-1)(k-1)-(2 k-1)|A|+k^{2} m \\
= & k m-(2 k-1)|A|+k^{2}-k .
\end{aligned}
$$

\section{Proofs}

Proof of Theorem 1. Let $A$ be a given subset of $G$ such that $R_{A}(g) \leq 5$ for all $g \in G$. Then

$$
\begin{aligned}
\sum_{g \in G}\left(R_{A}(g)-3\right)^{2} & =9\left|S_{0}\right|+4\left|S_{1}\right|+\left|S_{2}\right|+\left|S_{4}\right|+4\left|S_{5}\right| \\
& \leq 8\left|S_{0}\right|+3\left(\left|S_{1}\right|+\left|S_{3}\right|+\left|S_{5}\right|\right)+\left(\left|S_{0}\right|+\left|S_{1}\right|+\left|S_{2}\right|+\left|S_{3}\right|+\left|S_{4}\right|+\left|S_{5}\right|\right) .
\end{aligned}
$$

It is clear that

$$
\left|S_{0}\right|+\left|S_{1}\right|+\left|S_{2}\right|+\left|S_{3}\right|+\left|S_{4}\right|+\left|S_{5}\right|=\left|\left\{g: g \in G, 0 \leq R_{A}(g) \leq 5\right\}\right|=m,
$$




$$
\left|S_{1}\right|+\left|S_{3}\right|+\left|S_{5}\right|=\left|\left\{g: g \in G, 2 \nmid R_{A}(g)\right\}\right|=|\{2 a: a \in A\}| \leq|A| .
$$

Hence we have

$$
\sum_{g \in G}\left(R_{A}(g)-3\right)^{2} \leq 8\left|S_{0}\right|+3|A|+m
$$

On the other hand, by Lemma 3, taking $k=3$, we have

$$
\sum_{g \in G}\left(R_{A}(g)-3\right)^{2} \geq 3 m-5|A|+6
$$

Therefore, by (11),(2) and Lemma 1) it follows that

$$
\left|S_{0}\right| \geq \frac{1}{4} m-|A|+\frac{3}{4} \geq \frac{1}{4} m-\sqrt{5 m} .
$$

Now we prove part (b). Let $p$ be a prime number and $m=2\left(p^{2}+p+1\right)$. By Lemma 2 , there is a set $B \subseteq \mathbb{Z}_{p^{2}+p+1}$ such that $R_{B,-B}(n)=1$ for all $n \in \mathbb{Z}_{p^{2}+p+1}$ and $n \neq \overline{0}$. Then for any integer $l$ with $0 \leq l \leq p^{2}+p$, we define

$$
A_{l}=2 B \cup(2 B+2 l+1) \bmod m, \quad \text { where } 2 B=\{2 b: b \in B\} .
$$

Now we first prove that $R_{A_{l}}(n) \leq 4$ for all $n \in \mathbb{Z}_{m}$.

If $2 \mid n$, then $n=a_{1}+a_{2}$ with $a_{1}, a_{2} \in 2 B$ or $a_{1}, a_{2} \in 2 B+2 l+1$. Hence $R_{A_{l}}(n)=$ $R_{B}\left(\frac{n}{2}\right)+R_{B}\left(\frac{n}{2}-(2 l+1)\right) \leq 2+2=4$.

If $2 \nmid n$, then $n=a_{1}+a_{2}$ with $a_{1} \in 2 B, a_{2} \in 2 B+2 l+1$ or $a_{1} \in 2 B+2 l+1, a_{2} \in 2 B$. Hence, $R_{A_{l}}(n)=R_{B}\left(\frac{n-2 l-1}{2}\right) \times 2 \leq 4$.

Therefore, $R_{A_{l}}(n) \leq 4$ for all $n \in \mathbb{Z}_{m}$.

Let $P$ be a statement and we define

$$
I(P)= \begin{cases}1, & \text { if the statement } P \text { is true; } \\ 0, & \text { if the statement } P \text { is false. }\end{cases}
$$

Let

$$
\begin{gathered}
X_{\text {odd }}^{l}=\left\{2 k+1: 2 k+1 \in \mathbb{Z}_{m} \text { and } R_{A_{l}}(2 k+1)=0\right\}, \\
X_{\text {even }}^{l}=\left\{2 k: 2 k \in \mathbb{Z}_{m} \text { and } R_{A_{l}}(2 k)=0\right\} .
\end{gathered}
$$

Then $S_{0}=X_{\text {odd }}^{l} \cup X_{\text {even }}^{l}$. It is clear that $R_{A_{l}}(2 n+1)=0$ if and only if $R_{B}(n-l)=0$. Then

$$
\begin{aligned}
\left|X_{\text {odd }}^{l}\right| & =p^{2}+p+1-\sharp\left\{n: n \in \mathbb{Z}_{p^{2}+p+1}, R_{B}(n)=2\right\}-\sharp\left\{n: n \in \mathbb{Z}_{p^{2}+p+1}, R_{B}(n)=1\right\} \\
& =p^{2}+p+1-\left(\begin{array}{c}
p+1 \\
2
\end{array}\right)-(p+1)=\frac{1}{2} p^{2}-\frac{1}{2} p<\frac{1}{4} m .
\end{aligned}
$$


and

$$
\begin{aligned}
\sum_{l=0}^{p^{2}+p}\left|X_{\text {even }}^{l}\right| & =\sum_{l=0}^{p^{2}+p} \mid\left\{n: n \in \mathbb{Z}_{p^{2}+p+1}, R_{B}(n)=0 \text { and } R_{B}(n-2 l-1)=0\right\} \mid \\
& =\sum_{l=0}^{p^{2}+p} \sum_{n=0}^{p^{2}+p} I\left(R_{B}(n)=0 \text { and } R_{B}(n-2 l-1)=0\right) \\
& =\sum_{l=0}^{p^{2}+p} \sum_{n=0}^{p^{2}+p} I\left(R_{B}(n)=0\right) I\left(R_{B}(n-2 l-1)=0\right) \\
& =\sum_{n=0}^{p^{2}+p} I\left(R_{B}(n)=0\right) \sum_{l=0}^{p^{2}+p} I\left(R_{B}(n-2 l-1)=0\right) \\
& =\left(\frac{p^{2}}{2}-\frac{p}{2}\right) \sum_{n=0}^{p^{2}+p} I\left(R_{B}(n)=0\right)=\left(\frac{p^{2}}{2}-\frac{p}{2}\right)^{2} .
\end{aligned}
$$

Hence there is an integer $l$ such that

$$
\left|X_{\text {even }}^{l}\right| \leq \frac{1}{4} \cdot \frac{\left(p^{2}-p\right)^{2}}{p^{2}+p+1}<\frac{1}{4}\left(p^{2}+p+1\right)=\frac{1}{8} m .
$$

Therefore, for this integer $l$,

$$
\left|S_{0}\right|=\left|X_{\text {odd }}^{l}\right|+\left|X_{\text {even }}^{l}\right|<\frac{1}{4} m+\frac{1}{8} m=\frac{3}{8} m .
$$

Proof of Theorem [2. By Lemma 3, taking $k=2$, we have

$$
\sum_{g \in G}\left(R_{A}(g)-2\right)^{2} \geq 2 m-3|A|+2 .
$$

On the other hand,

$$
\begin{aligned}
\sum_{g \in G}\left(R_{A}(g)-2\right)^{2} & =4\left|S_{0}\right|+\left|S_{1}\right|+\left|S_{3}\right|+4\left|S_{4}\right|+9\left|S_{5}\right| \\
& \leq 4\left(\left|S_{0}\right|+\left|S_{4}\right|\right)+9\left(\left|S_{1}\right|+\left|S_{3}\right|+\left|S_{5}\right|\right) \\
& \leq 4\left(\left|S_{0}\right|+\left|S_{4}\right|\right)+9|A| .
\end{aligned}
$$

Hence, by (3) and (4), we have $\left|S_{0}\right|+\left|S_{4}\right| \geq \frac{1}{2} m-3|A|+\frac{1}{2} \geq \frac{1}{2} m-3 \sqrt{5 m}$. Since

$$
\left|S_{0}\right|+\left|S_{1}\right|+\left|S_{2}\right|+\left|S_{3}\right|+\left|S_{4}\right|+\left|S_{5}\right|=m,
$$

it follows that

$$
\left|S_{2}\right| \leq \frac{1}{2} m+3 \sqrt{5 m}
$$

Now we prove the part (b). By Lemma 2, there exists a subset $A \subseteq \mathbb{Z}_{m}$ such that $R_{A,-A}(n)=1$ for all $n \in \mathbb{Z}_{m}, n \neq \overline{0}$. It is easy to see that $|A|=p+1$ and $R_{A}(n) \leq 2$ for all $n \in \mathbb{Z}_{m}$. Hence

$$
\left|S_{2}\right|=\left(\begin{array}{c}
|A| \\
2
\end{array}\right)=\frac{1}{2}\left(p^{2}+p+1\right)-\frac{1}{2}=\frac{1}{2} m-\frac{1}{2}
$$


Proof of Theorem 3. By Lemma 3, taking $k=4$, we have

$$
\sum_{g \in G}\left(R_{A}(g)-4\right)^{2} \geq 4 m-7|A|+12 .
$$

On the other hand, by $\left|S_{1}\right|+\left|S_{3}\right|+\left|S_{5}\right|+\left|S_{7}\right| \leq|A|$, we have

$$
\begin{aligned}
\sum_{g \in G}\left(R_{A}(g)-4\right)^{2} & =16\left|S_{0}\right|+9\left|S_{1}\right|+4\left|S_{2}\right|+\left|S_{3}\right|+\left|S_{5}\right|+4\left|S_{6}\right|+9\left|S_{7}\right| \\
& \leq 4\left(\left|S_{0}\right|+\left|S_{2}\right|+\left|S_{4}\right|+\left|S_{6}\right|\right)+9|A|+12\left|S_{0}\right|-4\left|S_{4}\right| \\
& \leq 4 m+9|A|+12\left|S_{0}\right|-4\left|S_{4}\right| .
\end{aligned}
$$

Hence $\left|S_{4}\right| \leq 4|A|+3\left|S_{0}\right|+3$. Since $\sum_{i=0}^{7}\left|S_{i}\right|=m$, it follows that

$$
m \geq\left|S_{0}\right|+\left|S_{4}\right| \geq \frac{\left|S_{4}\right|-4|A|-3}{3}+\left|S_{4}\right|=\frac{4}{3}\left|S_{4}\right|-\frac{4}{3}|A|-1 .
$$

By Lemma 1, we have

$$
\left|S_{4}\right| \leq \frac{3}{4} m+|A|+\frac{3}{4} \leq \frac{3}{4} m+\sqrt{7 m}+\frac{3}{4} .
$$

Now we prove the part (b). Let $p$ be a prime and $m=2\left(p^{2}+p+1\right)$. By Lemma 2 there exists a subset $A_{p} \subseteq \mathbb{Z}_{p^{2}+p+1}$ such that $R_{A_{p},-A_{p}}(n)=1$ for all $n \neq \overline{0}$. Let $A=2 A_{p} \cup\left(p^{2}+p+1+2 A_{p}\right) \subseteq \mathbb{Z}_{m}$.

If $2 \mid n$, then $R_{A}(n)=R_{A_{p}}\left(\frac{n}{2}\right)+R_{A_{p}}\left(\frac{n}{2}\right) \leq 2+2=4$. If $2 \nmid n$, then $R_{A}(n)=$ $2 R_{A_{p}}\left(\frac{n-\left(p^{2}+p+1\right)}{2}\right) \leq 4$. Hence $R_{A}(n) \leq 4$ for all $n \in \mathbb{Z}_{m}$.

$$
\begin{aligned}
\left|S_{4}\right| & =\mid\left\{n: 2 \mid n, n \in \mathbb{Z}_{m} \text { and } R_{A_{p}}\left(\frac{n}{2}\right)=2\right\} \mid \\
& +\mid\left\{n: 2 \nmid n, n \in \mathbb{Z}_{m} \text { and } R_{A_{p}}\left(\frac{n-\left(p^{2}+p+1\right)}{2}\right)=2\right\} \mid \\
& =\mid\left\{n: n \in \mathbb{Z}_{p^{2}+p+1} \text { and } R_{A_{p}}(n)=2\right\}|+|\left\{n: n \in \mathbb{Z}_{p^{2}+p+1} \text { and } R_{A_{p}}\left(n-\frac{p^{2}+p}{2}\right)=2\right\} \mid \\
& =2\left(\begin{array}{c}
p+1 \\
2
\end{array}\right)=p^{2}+p=\frac{1}{2} m-1 .
\end{aligned}
$$

\section{Acknowledgement}

This work was done during the second author visiting to Budapest University of Technology and Economics. He would like to thank Dr. Sándor Kiss and Dr. Csaba Sándor for their warm hospitality. 


\section{References}

[1] P. Borwein, S. Choi and F. Chu, An old conjecure of Erdös-Turán on additive bases, Math. Comp. 75 (2005), 475-484.

[2] Y.-G. Chen, The analogue of Erdös-Turán conjecture in $\mathbb{Z}_{m}$, J. Number Theory 128 (2008), 2573-2581.

[3] Y.-G. Chen, On the Erdős-Turán conjecture, C. R. Math. Acad. Sci. Paris 350 (2012), 933-935.

[4] Y.-G. Chen and T. Sun, The difference basis and bi-basis of $\mathbb{Z}_{m}$, J. Number Theory 130 (2010), 716-726.

[5] A. Dubickas, A basis of finite and infinite sets with small representation, The Electronic J. Combin. 19 (2012), R6.

[6] P. Erdős, On a problem of Sidon in additive number theory, Acta Sci. Math. (Szeged) 15 (1954), 255-259.

[7] P. Erdős and P. Turán, On a problem of Sidon in additive number theory, and on some related problems, J. Lond. Math. Soc. 16 (1941), 212-215.

[8] G. Grekos, L. Haddad, C. Helou and J. Pihko, On the Erdôs-Turán conjecture, J. Number Theory 102 (2003), 339-352.

[9] L. Haddad and C. Helou, Bases in some additive groups and the Erdős-Turán conjecture, J. Combin. Theory Ser. A 108 (2004), 147-153.

[10] L. Haddad and C. Helou, Additive bases representations in groups, Integers 8 (2008), A5, 9 pp.

[11] I. Konstantoulas, Lower bounds for a conjecture of Erdös and Turán, Acta Arith. 159 (2013), 301-313.

[12] S. V. Konyagin and V. F. Lev, The Erdös-Turán problem in infinite groups, Additive number theory, 195-202, Springer, New York, 2010.

[13] V. F. Lev and A. Sárközy, An Erdös-Fuchs type theorem for finite groups, Integers 11(4) (2012), 487-494 .

[14] Y.-L. Li and Y.-G. Chen, On additive representation functions, to appear in Bull. Aust. Math. Soc., doi:10.1017/S0004972717000302.

[15] M. B. Nathanson, Unique representation bases for integers, Acta Arith. 108 (2003), 1-8. 
[16] I. Z. Ruzsa, A just basis, Monatsh. Math. 109 (1990), 145-151.

[17] C. Sándor and Q.-H. Yang, A lower bound of Ruzsa's number related to the Erdös-Turán conjecture, to appear in Acta Arith.

[18] J. Singer, A theorem in finite projective geometry and some applications to number theory, Trans. Amer. Math. Soc. 43 (1938) 377-385.

[19] M. Tang, On the Erdős-Turán conjecture, J. Number Theory 150 (2015), 74-80.

[20] Q.-H. Yang, A generalization of Chen's theorem on the Erdös-Turán conjecture, Int. J. Number Theory 9 (2013), 1683-1686. 